\title{
Trp53 Mutation in Keratin 5 (Krt5)-Expressing Basal Cells Facilitates the Development of Basal Squamous-Like Invasive Bladder Cancer in the Chemical Carcinogenesis of Mouse Bladder
}

Norihiko Masuda, * Kaoru Murakami, * Yuki Kita, * Akihiro Hamada, * Mayumi Kamada, ${ }^{\dagger}$ Yuki Teramoto, ${ }^{\ddagger}$ Toru Sakatani, Keiyu Matsumoto, ${ }^{*}$ Takeshi Sano, ${ }^{*}$ Ryoichi Saito, ${ }^{*}$ Yasushi Okuno, ${ }^{\dagger}$ Osamu Ogawa, ${ }^{*}$ and Takashi Kobayashi*

From the Department of Urology, ${ }^{*}$ the Department of Biomedical Data Intelligence, ${ }^{\dagger}$ and the Department of Diagnostic Pathology, ${ }^{\ddagger}$ Kyoto University Graduate School of Medicine, Kyoto, Japan

Accepted for publication

April 8, 2020.

Address correspondence to Osamu Ogawa, M.D., Ph.D., Department of Urology, Kyoto University Graduate School of Medicine, 54

Shogoinkawahara-cho, Sakyoku, Kyoto 606-8507, Japan. Email: ogawao@kuhp.kyoto-u. ac.jp.

\begin{abstract}
The biological processes of urothelial carcinogenesis are not fully understood, particularly regarding the relationship between specific genetic events, cell of origin, and molecular subtypes of subsequent tumors. N-butyl-N-(4-hydroxybutyl)-nitrosamine (BBN)-induced mouse bladder cancer is widely accepted as a useful model that recapitulates the pathway of human bladder tumorigenesis from dysplasia to invasive cancer via carcinoma in situ. However, the long and variable time of tumorigenesis often hinders efficient preclinical or translational research. We hypothesized that Trp53 mutation in specific types of urothelial cells facilitates efficient development of clinically relevant bladder cancer. Using lineage tracing, we showed that Trp53 mutation in Krt5-expressing cells resulted in more efficient tumorigenesis of mouse muscle-invasive bladder cancer (MIBC) with squamous differentiation compared with Trp53 mutation in Upk2-expressing cells, or wild-type or hemizygous Trp53 in the entire urothelium. Mouse MIBC that developed at 24 weeks of BBN treatment showed morphologic and genetic similarities to the basal squamous subtypes of human MIBC, irrespective of pre-induction of Trp53 mutation or whether the cell of origin was Krt5- or Upk2-expressing cells. Our findings suggest that intermediate cells as well as basal cells also can give rise to basal-like MIBC, with pre-induction of Trp53 mutation accelerating MIBC. Thus, in BBN chemical carcinogenesis, pre-induction of Trp53 mutation in basal cells facilitates efficient modeling of the basal squamous subtype of human MIBC. (Am J Pathol 2020, 190: 1752-1762; https://doi.org/10.1016/j.ajpath.2020.04.005)
\end{abstract}

Bladder cancer is a major malignancy worldwide, with more than 80,000 estimated new cases per year in the United States. It causes more than 17,000 estimated mortalities, which accounts for $2.9 \%$ of all cancer mortalities. ${ }^{1}$ Most bladder cancer is classified pathologically as urothelial carcinoma. Non-muscle-invasive bladder cancer (MIBC) can be treated by transurethral resection with an excellent survival outcome. ${ }^{2}$ However, MIBC in most instances requires the total removal of the bladder and is associated frequently with regional lymph node involvement and distant metastasis.

The prognosis of patients with MIBC or metastatic urothelial carcinoma is not favorable and has not improved remarkably for decades. ${ }^{2}$ This largely may be because the molecular pathogenesis and biological processes of bladder carcinogenesis including cell of origin have not been fully understood. $^{3}$ Recent efforts by The Cancer Genome Atlas (TCGA) and others have shown the genetic landscape of MIBC, which led to the definition of molecular subtypes with treatment implications. ${ }^{4-11}$ Specifically, however, treatment of the basal squamous subtype of MIBC, which is accompanied frequently by squamous differentiation, is still

Supported by JSPS KAKENHI grants JP25713055 (T.K.), JP19H03790 (T.K.), and JP26253078 (O.O.).

K.M., Y.K., A.H., and M.K. contributed equally to this work.

Disclosures: None declared. 
a clinical challenge because it is associated with treatment resistance and unfavorable oncological outcomes. ${ }^{12,13}$

Thus, there is an urgent need to improve the biological understanding of the basal subtype of MIBC and develop novel treatment strategies. MIBC is one of the most understudied cancers, partly because of the lack of faithful disease models. ${ }^{14}$ Recently, N-butyl-N-(4-hydroxybutyl)-nitrosamine (BBN)-induced mouse bladder tumors have been shown to share its genetic landscape, including mutational signatures and gene alterations, with the basal subtype of human MIBC. ${ }^{15,16}$ Although another mouse model based on bladderspecific deletion of Trp53 and Pten tumor suppressors also was reported to have the gene expression signature of the basal subtype of human MIBC, ${ }^{17,18}$ the BBN mouse model is the most widely used mouse model for bladder cancer at present because of its technical ease and lower cost.

One of the shortcomings of BBN-induced bladder carcinogenesis as an animal model is the long time period required for the development of MIBC, reported as being 5 to 6 months, with a wide variation in the tumor progression rate. ${ }^{19,20}$ It is challenging to have animals develop bladder tumors quickly and constantly, hindering translational and preclinical studies. This seems to be owing to the high mutagenesis of BBN-derived carcinogens, resulting in exceedingly heterogeneous clones in each affected animal.

In addition, it has not been fully understood what type of cells in the urothelium give rise to the specific subtypes of bladder cancer. In this regard, Shin et $\mathrm{al}^{21}$ showed that Sonic hedgehog-expressing cells in the urothelium are the cell of origin of mouse bladder cancer and loss of Sonic hedgehog expression is associated with disease progression. In another report using lineage tracing methods, Van Batavia et al $^{22}$ showed that $U p k 2$-expressing intermediate cells gave rise to papillary bladder cancers, whereas $\mathrm{Krt5}$-expressing basal cells likely are progenitors of carcinoma in situ and muscle-invasive lesions.

Herein, we hypothesized that more efficient and lessvariable tumors could develop in the BBN mouse bladder cancer model by pre-inducing specific gene alterations in relevant types of urothelial cells. This research shows that pre-induction of Trp53 mutation in Krt5-expressing urothelial cells promotes the most efficient development of mouse MIBC, mimicking the basal squamous subtypes of human bladder cancer.

\section{Materials and Methods}

\section{Animal Experiments}

FVB-Tg(KRT5-cre/ERT2 $)^{A J d g / M m m h}\left(\right.$ Krt5 $\left.5^{\text {CreERT2 }}\right),{ }^{23}$ C57BL/ 6 (LSL-RFP) ${ }^{24} 129 S$-Trp5 $3^{\text {tm } 2 T y j J J}\left(\operatorname{Trp} 53^{R 172 H}\right),{ }^{25}$ and $129 S$ $\operatorname{Trp} 53^{\text {tm3Tyj/J }}\left(\operatorname{Trp} 53^{R 270 H}\right)^{25}$ strains were obtained from the Jackson Laboratory (Bar Harbor, ME). The TgUICBAC strain $\left(U p k 2^{\text {CreERT2 }}\right)^{26}$ was a kind gift from Dr. Carlos CordonCardo (Icahn School of Medicine at Mount Sinai, New York, NY). Upk2 $2^{\text {CreERT2 }}$ and $\mathrm{Krt5} 5^{\mathrm{CreERT2}}$ mice were bred with the LSL-red fluorescent protein (RFP) line and then crossed with the $\operatorname{Trp} 53^{R 172 H}$ or $\operatorname{Trp} 53^{R 270 H}$ strain $\left(\operatorname{Trp} 53^{m u t}\right)$. When treated with tamoxifen, $U p k 2^{\text {CreERT2 }} ; L S L$-RFP;Trp53 $3^{+/ m u t}$ and $K R T 5^{C r E E R T 2} ; L S L$-RFP;Trp $53^{+/ m u t}$ mice expressed RFP and mutant Trp53 in cells expressing Upk2 and Krt5, respectively, at the time of tamoxifen treatment and thereafter in their lineage of progeny. $U p k 2^{C r e E R T 2} ; L S L$-RFP;Trp $53^{+/+}$ and $K R T 5^{\text {CreERT2 }} ;$ LSL-RFP;Trp $53^{+/+}$mice express only RFP upon tamoxifen treatment (wild-type Trp53 control). $U p k 2^{\text {CreERT2 }} ;$ LSL-RFP;Trp $53^{+/ m u t}$ and $K R T 5^{\text {CreERT2 }} ;$ LSLRFP; $\operatorname{Trp} 53^{+/ m u t}$ mice without tamoxifen treatment act as hemizygous Trp53 controls $\left(\operatorname{Trp} 53^{+/-}\right)$.

The mice were housed in a specific pathogen-free room. Tamoxifen was injected intraperitoneally at a dose of $0.2 \mathrm{mg} / \mathrm{g}$ body weight for 3 days in 5-week-old male and female mice. At 8 weeks old, mice were given drinking water with or without $0.1 \% \mathrm{BBN}$. For lineage tracing tumor characterization experiments, mice were given BBN (Tokyo Kasei Kogyo, Tokyo, Japan) for 24 weeks. For tumor-prevention experiments, mice were administered BBN for 6 weeks (age, 8 to $14 \mathrm{wk}$ ) and randomized to be untreated (control) or treated with gefitinib (Selleck Chemicals, Houston, TX) or AZD4547 (Selleck Chemicals), a fibroblast growth factor receptor 3 inhibitor, for 4 weeks (age, 10 to $14 \mathrm{wk}$ ). Gefitinib (dose, $2.5 \mathrm{mg} /$ body weight) was injected intraperitoneally 5 days per week according to a previous report. ${ }^{27}$ AZD4547 (dose, $5 \mathrm{mg} / \mathrm{kg}$ ) was injected intraperitoneally every other day according to a previous report. ${ }^{28}$

At the end of the protocol, mice were euthanized using $\mathrm{CO}_{2}$ and dissected as described previously. ${ }^{29}$ Urinary bladders were weighed and cut in half. One half was fixed in $10 \%$ formalin at room temperature for 48 to 72 hours, transferred into phosphate-buffered saline, dehydrated in a graded alcohol series, and embedded in paraffin. The other half was snap frozen and subject to RNA extraction.

All mouse experiments were performed in accordance with the policies of the Fundamental Guidelines for Proper Conduct of Animal Experiment and Related Activities in Academic Research Institutions by the Ministry of Education, Culture, Sports, Science, and Technology of Japan. The experimental protocol was approved by the Animal Research Committee, Graduate School of Medicine, Kyoto University (MedKyo13551, MedKyo14316, MedKyo15290, MedKyo16161, MedKyo17207, and MedKyo18238).

\section{Pathologic Evaluation}

Paraffin sections were cut (5- $\mu$ m thick), dewaxed in xylene, and treated with an ethanol gradient. The sections were stained with hematoxylin and eosin or with antibodies against keratin 5 (Krt5) (Ab52635, 1:100; Abcam, Cambridge, UK), keratin 14 (Krt14) (Ab7800, 1:200; Abcam), forkhead box A1 (Foxa1) (Ab170933, 1:200; Abcam), and epidermal growth factor receptor (Ab52894, 1:200; Abcam).

For immunofluorescence, antigen retrieval was performed with citrate buffer ( $\mathrm{pH} \mathrm{6)}$ before primary antibody incubation at $4^{\circ} \mathrm{C}$ overnight. Sections were stained with primary 
antibodies against Upk (Ab78196, 1:200; Abcam), Krt5 (Ab52635, 1:100; Abcam), and RFP (Ab62341, 1:50; Abcam), and secondary antibodies Alexa Fluor-488 and Alexa Fluor-594 $(10 \mu \mathrm{g} / \mathrm{mL}$; Thermo Fisher Scientific, Paisley, UK) for 1 hour at room temperature. Slides were mounted with Mowiol 488 (Sigma-Aldrich, Dorset, UK) containing DAPI (Vectashield Mounting Medium with DAPI, H-1200; Vector Laboratories, Burlingame, CA). Images were taken on a Leica confocal laser microscope (TCS SP8; Leica Microsystems, Wetzler, Germany).

Histopathologic evaluation of the bladder tissues was performed in a blind fashion by one pathologist at Kyoto Histopathology Study Group, Inc., and by two trained urologists (N.M. and T.K.), based on the 2004 World Health Organization Classification of Bladder Tumors.

\section{RNA Extraction}

Total RNA was extracted using an RNeasy Mini kit (Qiagen, Hilden, Germany) from whole bladders and reversetranscribed into cDNA using a ReverTra Ace Quantitative RT-PCR Kit (Toyobo, Osaka, Japan). Synthesized cDNA was subjected to cDNA expression array or quantitative RT-PCR.

\section{Gene Expression Profiling}

Gene expression profiles of mice were analyzed using the Agilent SurePrint G3 Mouse GE 8x60K ver 2.0 platform (Agilent Technologies, Santa Clara, CA), and the raw signal data were processed using Agilent Feature Extraction software. The processed data were imported into R (R Foundation for Statistical Computing, Vienna, Austria), ${ }^{30}$ and background correlation, quantile normalization, and $\log 2$ transformation were performed with limma version $3.42 .{ }^{31}$ The average values were used for within-array replicate probes, and then the expression data sets were constructed for each combination with wild-type and phenotype.

Establishment of Gene Sets Using The Cancer Genome Atlas Urothelial Bladder Carcinoma (TCGA-BLCA) mRNA Expression Profiles

TCGA-BLCA studies identified expression subtypes for human MIBC, which have different clinical features and published their research in $2014^{4}$ and 2017. ${ }^{8}$ To investigate correlations with the human MIBC profile, gene sets that characterize these subtypes using TCGA-BLCA data were constructed. mRNA expression data and clinical information (expression subtype for each patient) of each publication were obtained from the National Cancer Institute Genomic Data Commons (https://gdc.cancer.gov/about-data/publications, last accessed November 12, 2019) and the FireBrowse database (http://firebrowse.org, last accessed November 12, 2019). Each mRNA expression data set was processed based on protocols described elsewhere. ${ }^{4,8}$ Then, using genes that can be mapped to homologous mouse genes obtained from the Mouse Genome Informatics database, ${ }^{32}$ hierarchal clustering with Ward's method and correlation distance was performed for each data set. Based on the criteria of the Jain and Dubes ${ }^{33}$ method, gene sets for the TCGA data set were defined from the clustering. The list of genes for each gene set are described in Supplemental Tables S1-S4.

\section{Gene Set Enrichment Analysis}

To compare mouse gene expression profiles with TCGA-BLCA expression subtype, Gene Set Enrichment Analysis (GSEA) was used. GSEA is a well-known approach to analyze microarray data by comparing gene expression levels in different statuses (eg, wild-type and mutant) and predefined gene sets (eg, cancer-related genes). In this study, GSEA of mice expression data sets were performed with the following parameters: gene set size maximum, 1000; minimum, 25; scoring schema: weighted, metrix: Diff_of_Classes against the constructed gene sets of TCGA using GSEA software version 3.0 (Broad Institute, Cambridge, MA). ${ }^{34,35}$

\section{Quantitative RT-PCR}

Quantitative RT-PCR was performed as reported elsewhere $^{36}$ using the following primers: Gapdh: forward, 5'-GCACAGTCAAGGCCGAGAAT-3'; reverse, 5'-GCCTTCTCCATGGTGGTGAA-3'; Fgfr3: forward, 5'-GCCTGCGTGCTAGTGTTCT-3'; reverse, 5'-TACCATCCTTAGCCCAGACCG-3'; Krt5: forward, 5' TCTGCCATCACCCCATCTGT-3'; reverse, $5^{\prime}$-CCTCCGC CAGAACTGTAGGA-3'; Krt14: forward, 5'-AGCGGCA AGAGTGAGATTTCT-3'; reverse, 5'-CCTCCAGGTTA TTCTCCAGGG-3'; Egfr: forward, 5'-GCCATCTGGGCCAAAGATACC-3'; reverse, 5'-GTCTTCGCATGAATA GGCCAAT-3'; Gata3: forward, 5'-CTCGGCCATTCGTACATGGAA- $3^{\prime}$; reverse, 5'-GGATACCTCTGCACCG TAGC- ${ }^{\prime}$. The real-time PCR reaction was performed using the 7300 Real-Time PCR System and RT-PCR reagents (Power SYBR Green PCR Master Mix; Applied Biosystems, Foster City, CA) according to the manufacturer's protocol. The PCR reactions were performed in triplicate; three independent experiments were performed using different sets of samples. Data were normalized relative to the expression level of Gapdh.

\section{Statistical Analysis}

The data are presented as means \pm SD unless otherwise indicated. A $t$-test was used for the comparison of numeric data, and the $\chi^{2}$ test or the Fisher exact test was used for contingency analyses. For quantitative RT-PCR, the gene expression levels in each group were compared with those in another group by the post hoc test protected least significant difference (Fisher). All statistical analyses were performed using commercially available software (SPSSII; SPSS Japan, Inc., Tokyo, Japan). All tests were two-sided and $P$ values less than 0.05 were considered statistically significant. 
A

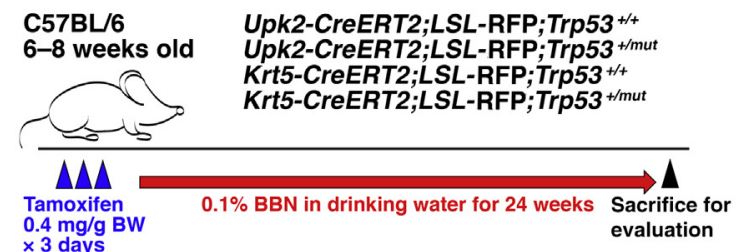

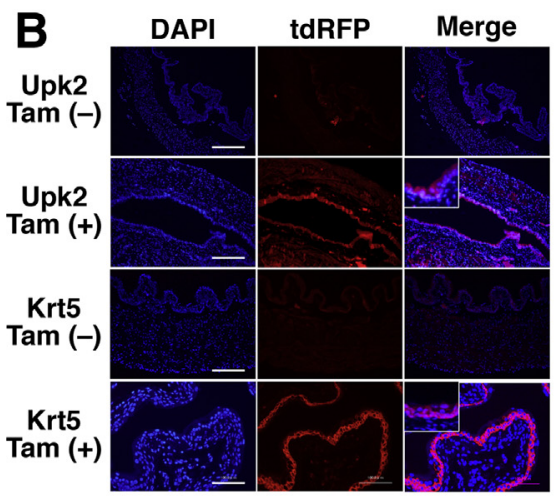

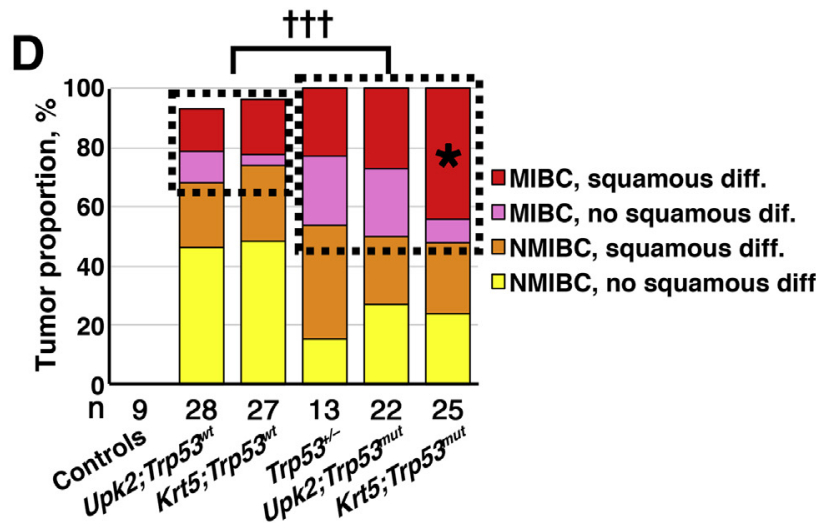

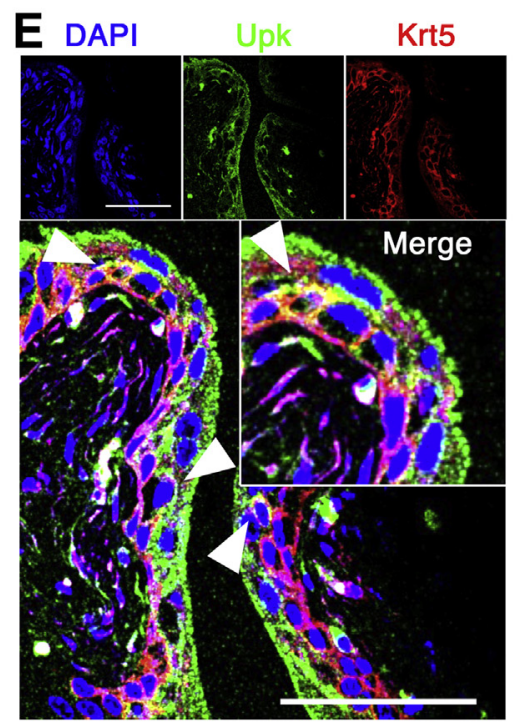

Figure 1 Induction of Trp53 mutation in keratin 5 (Krt5)-expressing cells promotes a higher incidence of muscle-invasive bladder cancer with squamous differentiation in the N-butyl-N-(4-hydroxybutyl)-nitrosamine (BBN)-induced murine bladder carcinogenesis model. A: Schematic design of the BBN-induced murine bladder carcinogenesis model. Six- to 8-week-old mice of the indicated genotypes were administered intraperitoneal tamoxifen followed by BBN treatment or no treatment for 24 weeks and then bladders were evaluated pathologically. B: Upk2-CreERT2;LSL-tandem dimer ( $t d$ ) red fluorescent protein (RFP) (Upk2) or Krt5-CreERT2;LSL-tdRFP (Krt5) mice were treated [Tam (+)] or untreated [Tam (-)] with tamoxifen, and then tdRFP expression was evaluated using fluorescence microscopy, showing that tdRFP is expressed in umbrella and intermediate cells in uroplakin 2 (Upk2) mice, while it is expressed in intermediate and basal cells in Krt5 mice. Higher magnification images are shown in the insets. C: Representative bladder tumors (arrowheads) in tdRFP-expressing mice receiving 24-week BBN treatment. D: Bars represent the proportion of muscleinvasive bladder cancer (MIBC) and non-MIBC (NMIBC) with or without squamous differentiation in the indicated genotypes of mice. None of the control mice formed bladder cancer. A subset of Upk2CreERT2;Trp53 $3^{+/+}$and Krt5-CreERT2;Trp53 $3^{+/+}$mice and $100 \%$ of Trp53 $3^{+/-}$, Upk2-CreERT2;Trp53 $3^{+/ m u t}$, and Krt5-CreERT2; Trp 53 $3^{+/ m u t}$ mice formed bladder cancer. Notably, Krt5-CreERT2; Trp 53 $3^{+/ m u t}$ mice developed MIBC with squamous differentiation most efficiently. E: Representative immunofluorescence images of the bladder from a wild-type untreated mouse. Note that a small subset of cells weakly express both Krt5 and Upk (arrowheads). $n=9$ control mice, $n=28$ Upk2-CreERT2;Trp53 ${ }^{+/+}$mice, $n=27 \mathrm{Krt5}$-CreERT2;Trp 53 $3^{+/+}$

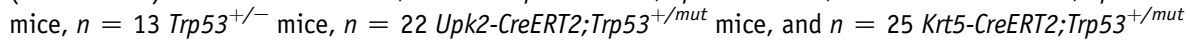
mice (D). ${ }^{*} P<0.05$ for Krt5; Trp53 ${ }^{\text {Mut }}$ versus others; ${ }^{\dagger \dagger \dagger} P<0.005$ frequency of MIBC between Trp53-wt mice and hemizygous/mutant Trp53 mice (dotted boxes). Scale bars: $100 \mu \mathrm{m}$ (B, C, and E). BW, body weight; diff., differentiation; H\&E, hematoxylin and eosin.

\section{Results}

\section{Monoallelic Trp53 Mutation Increases the Incidence of MIBC, Particularly in Krt5-Expressing Cells}

To investigate the cell of origin and the effect of $\operatorname{Trp} 53$ mutation in $\mathrm{BBN}$-induced chemical bladder carcinogenesis,
$0.4 \mathrm{mg} / \mathrm{g}$ per body weight of tamoxifen was administered to 6- to 8-week-old normal and transgenic mice for 3 days, which were then treated with $0.5 \% \mathrm{BBN}$ in drinking water for 24 weeks (Figure 1A). Upon tamoxifen treatment, tandem-dimer RFP with or without Trp53 mutation (R172H or $\mathrm{R} 270 \mathrm{H}$ ) was induced in $U p k 2$ - or $K r t 5$-expressing cells. Tandem-dimer RFP expression was confirmed in superficial 


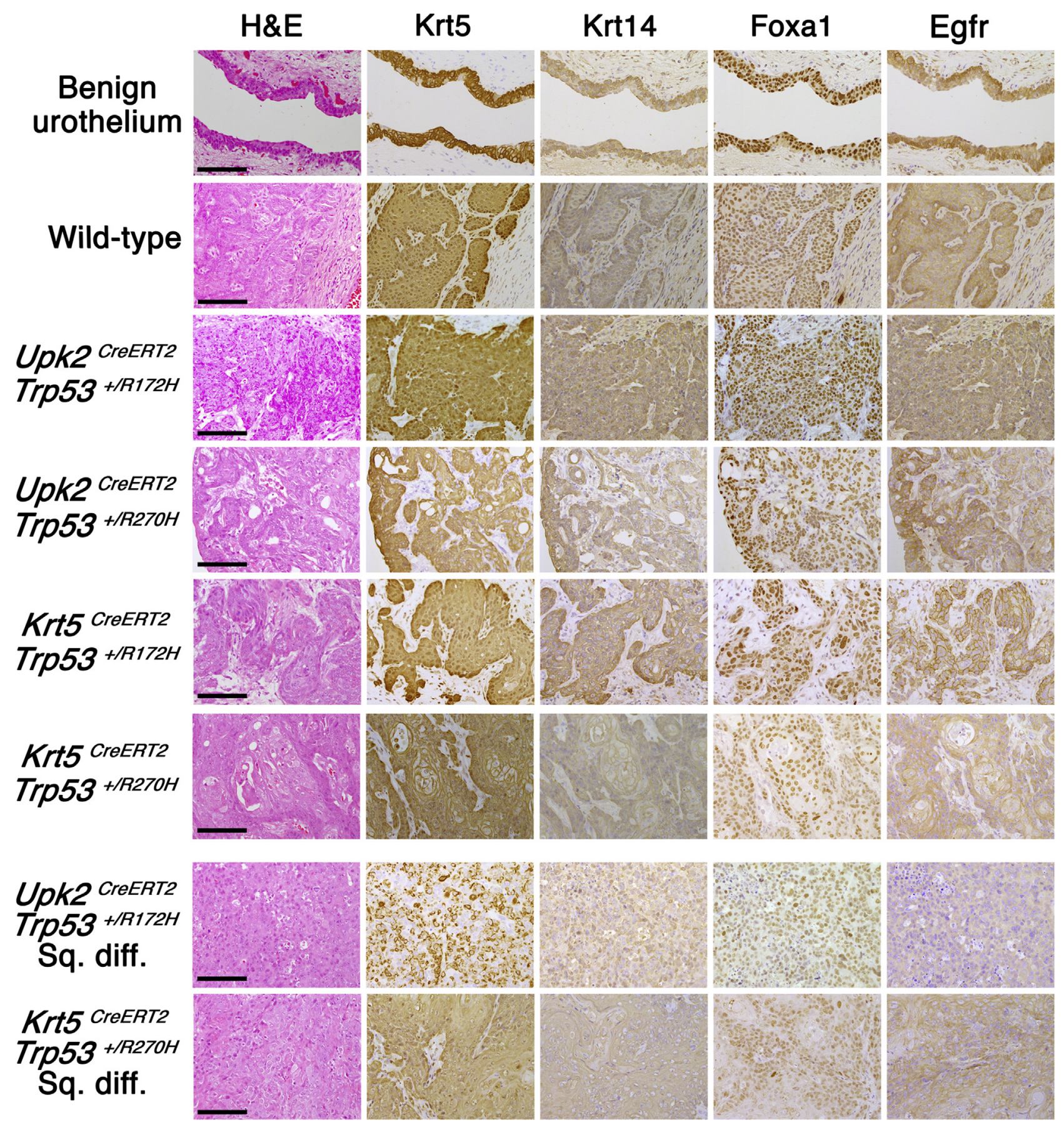

Figure 2 Expression of molecular subtype markers for urothelial carcinoma in N-butyl-N-(4-hydroxybutyl)-nitrosamine (BBN)-induced mouse bladder cancers. Serial sections from bladder specimens corresponding to normal bladder and tumors obtained from mice with the indicated genotypes were stained for previously reported (Material and Methods) markers of molecular subtypes of urothelial carcinoma, as indicated. Scale bars: $100 \mu \mathrm{m}$. Sq. diff., squamous differentiation; Egfr, epidermal growth factor receptor; Foxa1, forkhead box A1; H\&E, hematoxylin and eosin; Krt5, keratin 5; Krt14, keratin 14.

to intermediate layers of bladder urothelium in Upk2-RFP mice and intermediate to basal layers in Krt5-RFP mice 3 days after tamoxifen treatment, but not vehicle treatment (Figure 1B).

Mice were euthanized 24 weeks after BBN treatment, and the bladder was evaluated microscopically for RFPexpressing urothelial tumors (Figure 1C). Almost all mice developed urothelial carcinomas after 24 weeks of BBN treatment, but not after vehicle treatment (control) (Figure 1D). Mice in which a Trp53 mutation was not induced showed a higher rate $(40 \%$ to $50 \%)$ of non-MIBC without squamous differentiation. Mice in which monoallelic Trp53 mutation was induced or those harboring hemizygous loss of $\operatorname{Trp} 53$ showed a higher rate ( $40 \%$ to $50 \%$ ) of MIBCs. The differences in the rate of MIBCs were statistically significant (tumors with mutant/hemizygous versus wild-type 

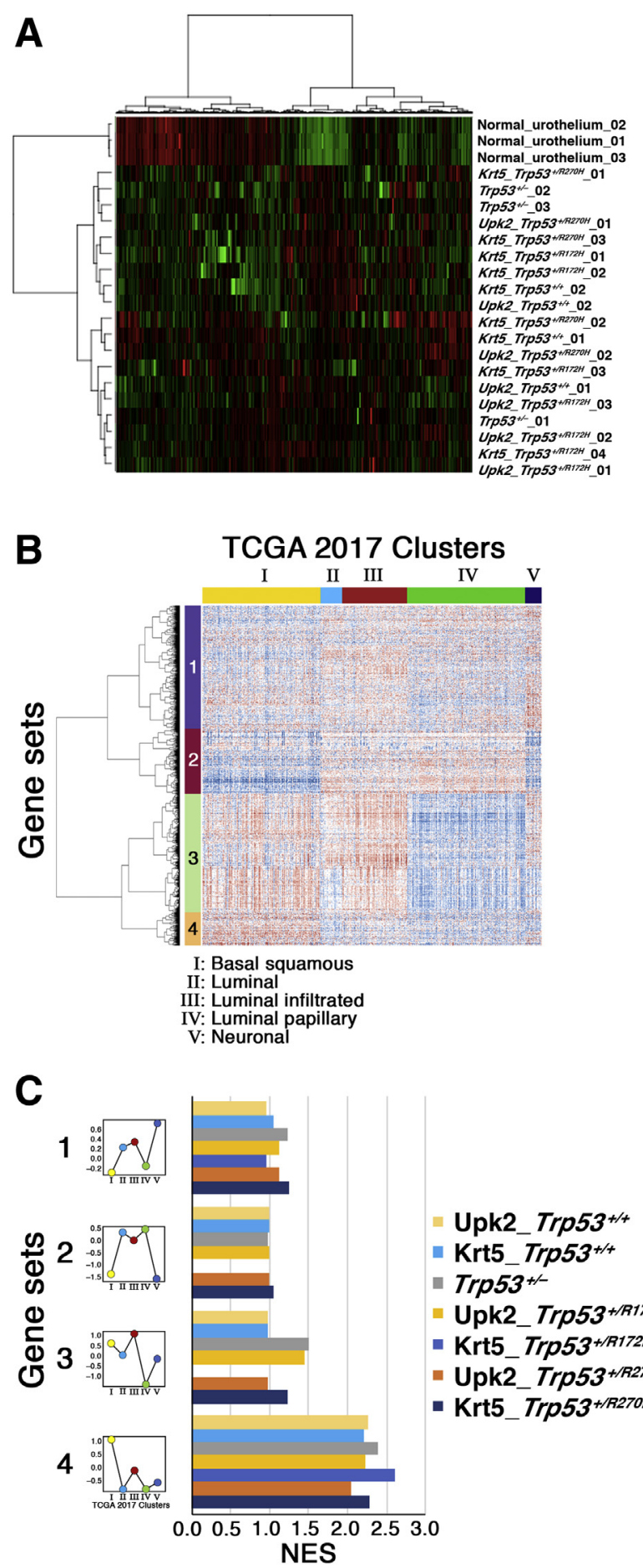

Figure 3 N-butyl-N-(4-hydroxybutyl)-nitrosamine (BBN)-induced murine urothelial carcinomas harbor molecular characteristics of the basal squamous subtype of human muscle-invasive bladder cancer irrespective of cell of origin or pre-induced Trp53 mutation. A: Nonhierarchical clustering of normal bladder urothelium and BBN-induced mouse bladder tumors from the indicated genotypes of mice. B: Hierarchical clustering based on The Cancer Genome Atlas (TCGA) $2017^{8}$ gene expression profile identified five gene sets with distinct expression patterns for each TCGA 2017 cluster. C: Left panels: Mean expression levels for indicated gene sets in the TCGA 2017 clusters. Right panel: Chart for normalized enrichment scores (NES) in the gene set enrichment analysis of bladder cancers from the indicated genotypes of mice with regard to normal bladder urothelium.
Trp53: $50 \%$ versus $24 \%, P=0.0035$, Fisher exact test; tumors with mutant versus wild-type Trp53: 51\% versus $24 \%, P=0.0041$, Fisher exact test). Notably, mice in which monoallelic Trp53 mutation was induced in Krt5expressing cells showed more than a twofold higher incidence $(>40 \%)$ of MIBC with squamous differentiation $\left(P=0.0145, \chi^{2}\right.$ test $)$.

This study investigated whether there was some overlap of Upk2- and Krt5-expressing cells in bladder urothelium using immunofluorescence. A subset of intermediate cells expressed both Upk2 and Krt5 (Figure 1E). Taken together, these results indicated that both $U p k 2$ - and $K r t 5$-expressing cells in the bladder urothelium can give rise to BBNinduced bladder cancer. Pre-induction of Trp53 mutation promoted muscle-invasive progression, and particularly, $\operatorname{Trp} 53$ mutation in Krt5-expressing cells most efficiently facilitated the development of MIBC with squamous differentiation.

BBN-Induced Tumors Harbor Molecular Characteristics of the Basal Squamous Subtype of Human Urothelial Carcinoma Irrespective of Background Genotype

Next, it was examined whether the pathologic and molecular characteristics differed between tumors derived from mice harboring distinct genotypes and Trp53 status. The immunohistochemical characteristics of $\mathrm{BBN}$-induced bladder cancers arising from each genotype of mice were investigated, while untreated benign bladder urothelium was evaluated as a control. Most BBN-induced MIBC showed strong expression of $\mathrm{Krt5}$ and stochastic expression of $\mathrm{Krt14}$, which was reported to be a characteristic of the basal squamous subtypes of human MIBC, ${ }^{4-11}$ consistent with previous reports (Figure 2). ${ }^{15,16}$ These tumors also were focally positive for Foxa1, which is considered a molecular marker of the luminal subtype of MIBC, ${ }^{4-11}$ with supporting functional rationales. ${ }^{37} \mathrm{BBN}$-induced mouse muscle-invasive urothelial carcinoma showed moderate to strong submembranous expression of epidermal growth factor receptor. However, these characteristics were not observed in lesions with squamous differentiation, except for Krt5, which still was moderately positive in those lesions.

The genetic characteristics of the mouse tumors were then analyzed. First, it was investigated whether mouse BBN-induced bladder cancers showed distinct gene expression profiles according to the cell of origin or pre-induced Trp53 mutation. Gene expression profiles based on cDNA microarray data were analyzed using nonhierarchical clustering, which showed that none of the mouse bladder tumors were clustered differentially based on the genotypes (Figure 3A, data are available for GSE147709). 

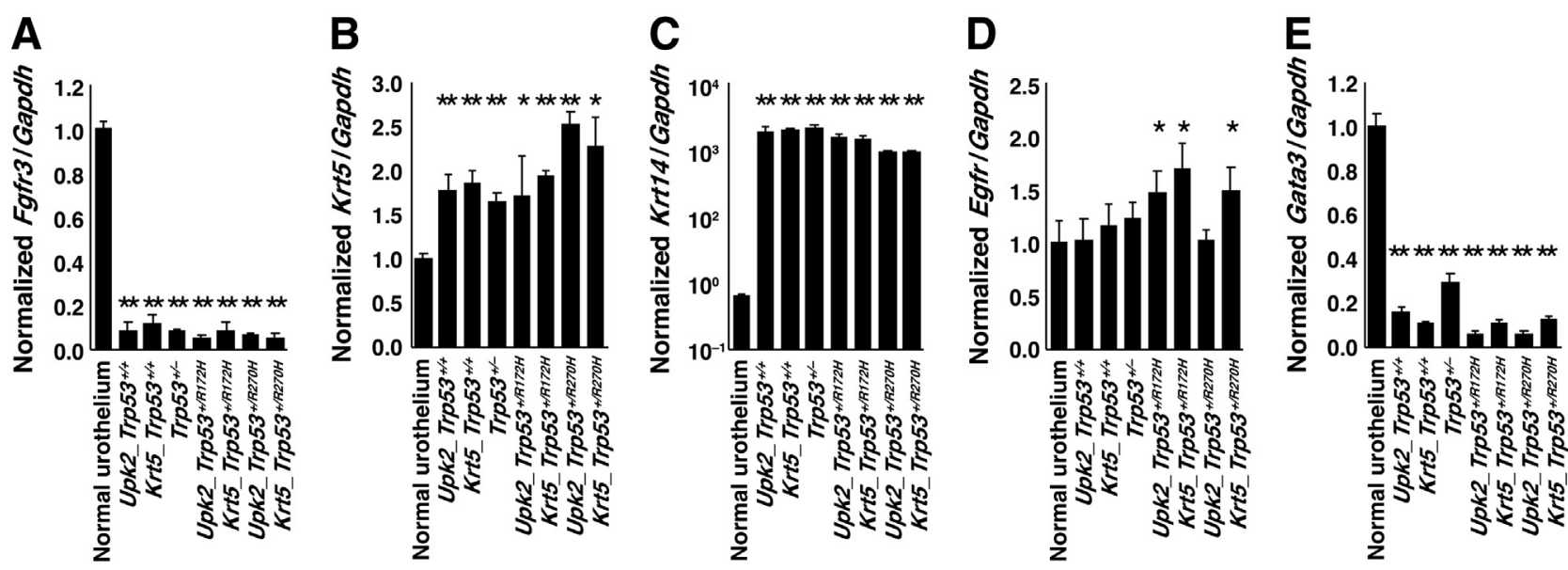

Figure 4 Chart for normalized expression of Fgfr3 (A), Krt5 (B), Krt14 (C), Egfr (D), and Gata3 (E) normalized against that of Gapdh for N-butyl-N-(4hydroxybutyl)-nitrosamine (BBN)-induced bladder tumors from the indicated genotypes of mice assessed by quantitative RT-PCR. ${ }^{*} P<0.05,{ }^{* *} P<0.01$ versus normal.

It is known that human MIBC is a molecularly diverse disease, and several molecular classifications have been proposed. ${ }^{4-11}$ This investigation evaluated whether our mouse MIBCs had molecular similarities to any subtypes of human MIBC. To this end, we decided to analyze the expression profiles of human MIBC by TCGA $2014^{4}$ and TCGA $2017^{8}$ because they are the most comprehensive molecular characterizations integrating somatic DNA alterations, chromosomal rearrangements, mRNA, miRNA, and protein expression. Because of these characteristics, TCGA data sets are expected to be used as the reference standard, ${ }^{9}$ even after the achievement of a consensus classification ${ }^{38}$ and a more recent publication of a newly developed classification based on a larger sized cohort. ${ }^{11}$ Specifically, the basal squamous subtype (cluster I in TCGA 20147), which is nearly correspondent to cluster 3 (so-called basal squamous-like subtype) in TCGA 2014, is characterized by its robust existence regardless of subtyping classifications, and is associated with unfavorable patient survival (Supplemental Figure S1A).

Reconciling hierarchical clustering data, gene sets that were enriched in specific patterns for each cluster of TCGA 2014 (Supplemental Figure S1, B and C) and 2017 (Figure 3, B and C, Supplemental Figures S2 and S3, Supplemental Tables $\mathrm{S} 1-\mathrm{S} 4$ ) were first generated. The optimal number of gene sets was determined as 6 and 13 for TCGA 2014, and as 4 and 11 for TCGA 2017 based on the Jain and Dubes ${ }^{33}$ method. Using these gene sets, GSEA was performed, which showed that all genotypes of $\mathrm{BBN}$ induced mouse bladder cancer were highly enriched in the specific gene sets to the basal squamous subtypes in both TCGA 2014 (Supplemental Figures S1C and S2) and 2017 (Figure 3C, Supplemental Figure S3), with a normalized enrichment score of approximately 2.5 , which is consistent with the immunohistochemical findings (Figure 2). The results of GSEA were supported further by quantified RTPCR, which showed down-regulated Fgfr3 and Gata3, and up-regulated $\mathrm{Krt5}, \mathrm{Krt14}$, and Egfr in the tumors compared with normal urothelium (Figure 4). These findings collectively suggest that BBN-induced mouse bladder cancer has high similarities in its genotypes and phenotypes to the basal squamous subtype of human MIBC, which is associated with poor patient survival.

Egfr Inhibition Suppresses the Development of MIBC with Squamous Differentiation in the BBN Mouse Chemical Carcinogenesis Model

Because the basal squamous subtype of bladder cancer is characterized by high epidermal growth factor receptor expression, and this characteristic is preserved in $\mathrm{BBN}$ induced mouse bladder cancer, this study investigated whether BBN-induced bladder carcinogenesis could be prevented by epidermal growth factor receptor inhibition. For this purpose, Krt5-CreERT2;LSL-RFP;Trp $53^{+/ L S L-m u t}$ mice were used because this genotype results in the most efficient MIBC with squamous differentiation. The mice were administered tamoxifen as described above (Animal Experiments) to induce Trp53 mutation and RFP reporter in $K r t 5$-expressing cells and then given $\mathrm{BBN}$ in drinking water for 6 weeks (Figure 5A). Then, after 2 weeks of BBN treatment, the mice were randomized to treatment with gefitinib, an epidermal growth factor receptor inhibitor. As the negative controls, other subsets of mice were treated with AZD4547, a fibroblast growth factor receptor inhibitor, or vehicle. After 4 weeks of treatment, there was no significant difference in bladder weight among the treatment groups (Figure 5B). Although there were no significant differences in the proportion of infiltrating ( $\mathrm{T} 1$ or higher) tumors (Figure 5C) or high-grade disease (Figure 5D), mice treated with gefitinib showed a significant decrease in tumors with squamous differentiation compared with vehicletreated mice (Figure 5E). 


\section{A Krt5-CreERT2;LSL-RFP;Trp53+LLL-mut} 5-week-old female mice

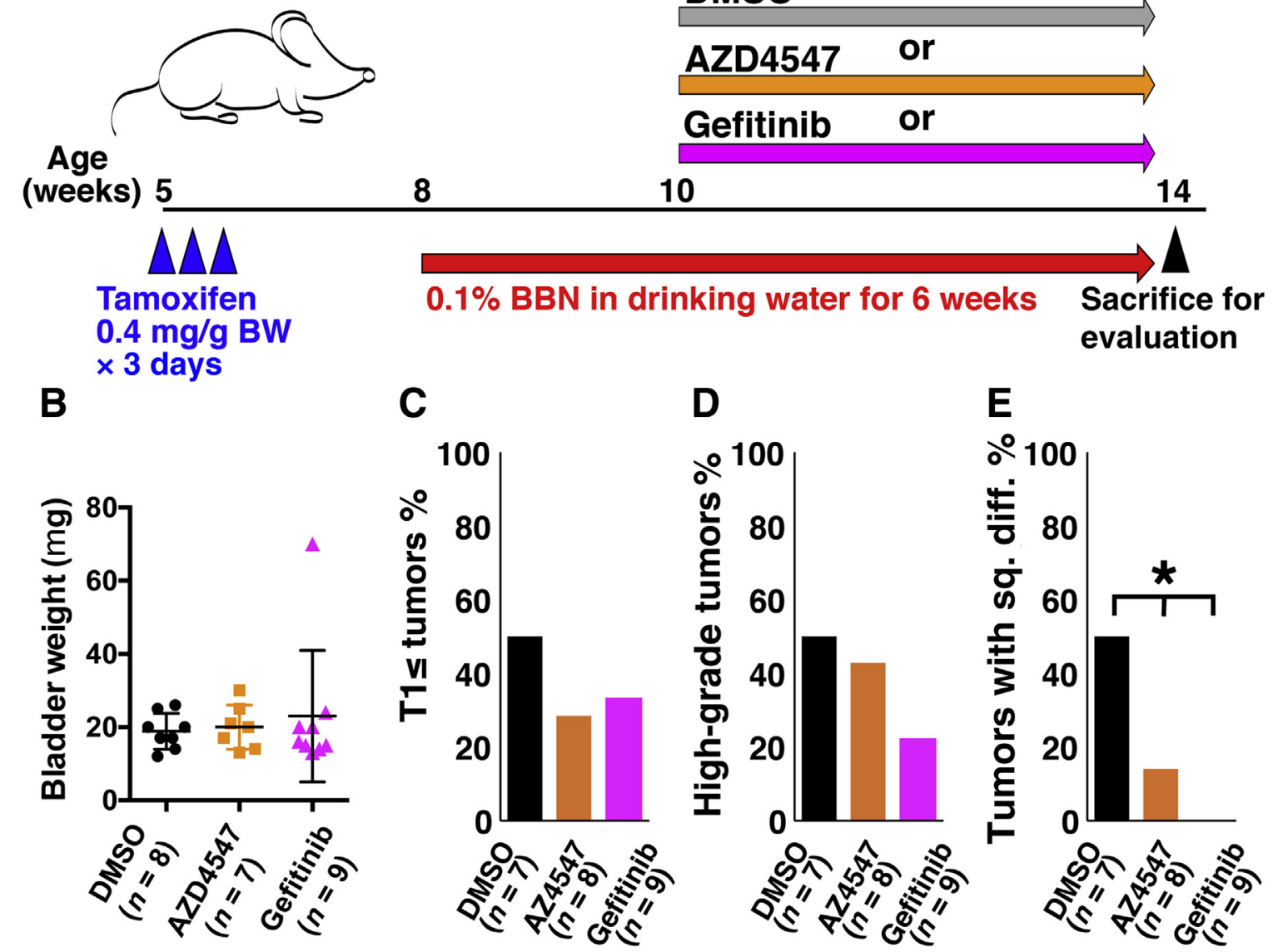

Figure 5 Pharmacologic inhibition of epidermal growth factor receptor (Egfr) suppresses the development of high-grade tumors with squamous differentiation (sq. diff.) in N-butyl-N-(4-hydroxybutyl)-nitrosamine (BBN)-induced murine bladder carcinogenesis in the context of mutant Trp53. A: Schematic design of the experiment. Krt5-CreERT2; Trp $53^{+/ m u t}$ mice were given $0.4 \mathrm{mg} / \mathrm{g}$ (body weight) intraperitoneal tamoxifen for 3 days at 5 weeks of age, followed by treatment with $0.1 \%$ BBN in drinking water from 8 weeks of age for 6 weeks. Then, mice were randomized to receive treatment with vehicle [dimethyl sulfoxide (DMS0)], fibroblast growth factor receptor inhibitor AZD4547, or EGFR inhibitor gefitinib from 10 weeks of age until the sixth week of BBN treatment. B: Bladder weight of each group of mice treated with the indicated drugs. C-E: Proportion of T1 or higher stage tumors (C), high-grade tumors (D), and tumors with squamous differentiation (E) for each group of mice treated with the indicated drugs. ${ }^{\star} P<0.05$ by $\chi^{2}$ test. BW, body weight.

\section{Discussion}

The present study showed that induction of $\operatorname{Trp} 53$ mutation in Krt5-expressing cells in the urothelium yields a high proportion of MIBC with squamous differentiation, which corresponds to the basal squamous subtypes of human MIBC. These findings provide useful information for bladder cancer modeling as well as having clinical and pathologic implications. The basal squamous subtypes of MIBC have been reported to be the most aggressive clinically and are associated with unfavorable clinical outcomes, ${ }^{12,13}$ as also shown in the present study based on reconciled TCGA data. It was implicated that the gene expression signature also is related to the pathologic classification or drug response of human bladder cancer. ${ }^{8,39-41}$ A similar correlation between the molecular subtypes and treatment response has been reported for bladder cancer cell lines, ${ }^{42}$ but has not yet been shown extensively in vivo using experimental animal models.
Therefore, modeling of this subtype of MIBC is highly relevant to clinically unmet needs and unanswered challenges.

In this regard, several investigators reported that BBNinduced murine bladder tumors share molecular characteristics with the basal squamous subtypes of human MIBC. ${ }^{15,16}$ The present study confirmed this more extensively by GSEA using newly developed gene sets showing molecular subtypespecific expression patterns. These gene sets will be useful resources for future GSEA or quick molecular subtyping in either human or mouse bladder cancers, benefitting both researchers and clinicians.

The low efficiency and wide variation of MIBC development in the BBN carcinogen-induced model has been its major inconsistency as an animal model for use in preclinical or translational investigations. These findings suggest that pre-induced Trp53 mutations predisposes urothelial cells to MIBC, which facilitates the development of BBNinduced bladder cancer more efficiently and consistently. 
TP53 is the most frequently altered gene in human MIBC, particularly in the basal squamous subtypes. In TCGA 2014, for example, TP53 alterations were detected in $86 \%$ of basal squamous-like cluster 3 tumors, and they were detected in $27 \%$ of the other subtypes $(P<0.0001$, Fisher exact test $)$. In TCGA 2017, TP53 alterations were detected in $60 \%$ of basal squamous tumors, and they were detected in $52 \%$ of the other subtypes $(P<0.0001$, Fisher exact test). These findings indicate that pre-induction of Trp53 would not make the mouse model deviate from the basal subtypes of human MIBC. The majority of tumors developed in Trp53 wild-type mice may harbor spontaneous $\operatorname{Trp} 53$ mutation or equivalent gene alterations because all muscle-invasive tumors from those mice also were categorized to the basal/ squamous subtype. Time to occurrence of $\operatorname{Trp} 53$ mutation may affect variable time to MIBC formation in mice with wild-type Trp53.

The present study has shown that both Upk2- and Krt5expressing cells can give rise to $\mathrm{BBN}$-induced MIBC in mice. This suggests the ability of intermediate cells, as well as basal cells, to originate from the basal subtype of MIBC and indicates the biological complexity of the pathogenesis and molecular subtypes based on gene mutation and expression profiles. Because the progeny of $\mathrm{Krt5}$-expressing cells develop MIBC with squamous differentiation more efficiently compared with those of $U p k 2$-expressing cells, it is suggested that basal cells are the major source of the basal squamous subtypes of MIBC. This can be attributed to the larger number of basal cells ${ }^{43}$ or the higher tumorigenic potential of basal cells than intermediate cells, which was shown in a previous report. ${ }^{44}$

According to a previous study using a lineage tracing method, Upk2-expressing intermediate cells gave rise to papillary bladder cancers, whereas $\mathrm{Krt5}$-expressing basal cells are likely to be progenitors of carcinoma in situ and muscle-invasive lesions. ${ }^{22}$ In the present study, the progeny of $U p k 2$-expressing cells also seemed to give rise to muscleinvasive lesions, whereas typical papillary lesions scarcely were observed in BBN-induced mouse bladder cancers, irrespective of genotype. This discrepancy might be owing to the difference in the Krt5-CreERT2 strain used in the present and previous studies. ${ }^{22}$ It also is possible that the relatively longer treatment with BBN in the present study allowed the Upk2-progenitor tumors to progress to muscleinvasive disease or allowed muscle-invasive lesions to become dominant. In addition, pre-induction of Trp53 mutations might promote the development of MIBC with squamous differentiation because $\operatorname{Trp} 53$ mutations are associated significantly with the basal squamous subtypes of MIBC (Figure 3). Moreover, enrichment of squamous differentiation would enable clinically relevant translational research that addresses clinically unmet needs because squamous differentiation was reported to be associated with unfavorable clinical outcomes. ${ }^{12,13}$

Immunohistochemical analysis showed the expression of luminal markers such as Foxa1 in BBN-induced tumors, even though the tumors were categorized genetically as basal squamous subtypes. This is often the case in human bladder cancers. Even when a tumor is largely positive for the basal marker KRT5, a subset of tumor cells can be positive for FOXA1. A recent report showed that human bladder cancers, particularly the basal squamous subtypes of MIBC, harbor intratumoral molecular heterogeneity. ${ }^{45}$

There were several limitations in this study that should be acknowledged. Because the expression profiles of differential markers in the three urothelial layers, as well as the proportion of the number of urothelial cells in these layers, are different between mice and humans, the findings of the study may not be directly applicable to human bladder carcinogenesis. In addition, phenotypic differences were not observed between the two Trp53 mutations, as reported previously, ${ }^{25}$ which should be investigated more profoundly in a future study. It would have been more informative if tumor formation had been monitored using ultrasound, which unfortunately was not performed in the present study. Nonetheless, this strategy of pre-inducing Trp53 mutations in Krt5-expressing cells before BBN treatment showed promising results in terms of enrichment and robustness in the development of the basal squamous subtypes of MIBC, which may be an advantage for various bladder cancer studies.

\section{Acknowledgments}

We thank Drs. Carlos Cordon-Cardo and Mireia CastilloMartin from the Icahn School of Medicine at Mount Sinai, New York, NY, for sharing the TgUICBAC strain $\left(U p k 2^{C r E E R T 2}\right)$ and for their help with revising the draft of this manuscript; Dr. Hideki Yokoi from the Kyoto University Graduate School of Medicine, Kyoto, Japan, for sharing the FVB-Tg(KRT5-cre/ERT2 $)^{A J d g / M m m h} \quad\left(K r t 5^{\text {CreERT2 }}\right)$ mouse; all past and present laboratory members of the Department of Urology, Kyoto University Graduate School of Medicine, Kyoto, Japan, for their technical assistance and intellectual support; and H. Nikki March, PhD, from Edanz Group (Fukuoka, Japan) for editing a draft of this manuscript.

\section{Supplemental Data}

Supplemental material for this article can be found at http://doi.org/10.1016/j.ajpath.2020.04.005.

\section{References}

1. Siegel RL, Miller KD, Jemal A: Cancer statistics, 2018. CA Cancer J Clin 2018, 68:7-30

2. Kamat AM, Hahn NM, Efstathiou JA, Lerner SP, Malmstrom PU, Choi W, Guo CC, Lotan Y, Kassouf W: Bladder cancer. Lancet 2016, 388:2796-2810

3. Knowles MA, Hurst CD: Molecular biology of bladder cancer: new insights into pathogenesis and clinical diversity. Nat Rev Cancer 2015, 15:25-41 
4. Cancer Genome Atlas Research Network: Comprehensive molecular characterization of urothelial bladder carcinoma. Nature 2014, 507: 315-322

5. Choi W, Porten S, Kim S, Willis D, Plimack ER, Hoffman-Censits J, Roth B, Cheng T, Tran M, Lee IL, Melquist J, Bondaruk J, Majewski T, Zhang S, Pretzsch S, Baggerly K, Siefker-Radtke A, Czerniak B, Dinney CP, McConkey DJ: Identification of distinct basal and luminal subtypes of muscle-invasive bladder cancer with different sensitivities to frontline chemotherapy. Cancer Cell 2014, 25:152-165

6. Damrauer JS, Hoadley KA, Chism DD, Fan C, Tiganelli CJ, Wobker SE, Yeh JJ, Milowsky MI, Iyer G, Parker JS, Kim WY: Intrinsic subtypes of high-grade bladder cancer reflect the hallmarks of breast cancer biology. Proc Natl Acad Sci U S A 2014, 111: $3110-3115$

7. Rebouissou S, Bernard-Pierrot I, de Reynies A, Lepage ML, Krucker C, Chapeaublanc E, Herault A, Kamoun A, Caillault A, Letouze E, Elarouci N, Neuzillet Y, Denoux Y, Molinie V, Vordos D, Laplanche A, Maille P, Soyeux P, Ofualuka K, Reyal F, Biton A, Sibony M, Paoletti X, Southgate J, Benhamou S, Lebret T, Allory Y, Radvanyi F: EGFR as a potential therapeutic target for a subset of muscle-invasive bladder cancers presenting a basal-like phenotype. Sci Transl Med 2014, 6:244ra91

8. Robertson AG, Kim J, Al-Ahmadie H, Bellmunt J, Guo G, Cherniack $\mathrm{AD}$, et al: Comprehensive molecular characterization of muscle-invasive bladder cancer. Cell 2017, 171:540-556.e25

9. Marzouka NA, Eriksson P, Rovira C, Liedberg F, Sjodahl G, Hoglund M: A validation and extended description of the Lund taxonomy for urothelial carcinoma using the TCGA cohort. Sci Rep 2018, 8:3737

10. Mo Q, Nikolos F, Chen F, Tramel Z, Lee YC, Hayashi K, Xiao J, Shen J, Chan KS: Prognostic power of a tumor differentiation gene signature for bladder urothelial carcinomas. J Natl Cancer Inst 2018, 110:448-459

11. Tan TZ, Rouanne M, Tan KT, Huang RY, Thiery JP: Molecular subtypes of urothelial bladder cancer: results from a meta-cohort analysis of 2411 tumors. Eur Urol 2019, 75:423-432

12. Black PC, Brown GA, Dinney CP: The impact of variant histology on the outcome of bladder cancer treated with curative intent. Urol Oncol 2009, 27:3-7

13. Minato A, Fujimoto N, Kubo T: Squamous differentiation predicts poor response to cisplatin-based chemotherapy and unfavorable prognosis in urothelial carcinoma of the urinary bladder. Clin Genitourin Cancer 2017, 15:e1063-e1067

14. Kobayashi T, Owczarek TB, McKiernan JM, Abate-Shen C: Modelling bladder cancer in mice: opportunities and challenges. Nat Rev Cancer 2015, 15:42-54

15. Saito R, Smith CC, Utsumi T, Bixby LM, Kardos J, Wobker SE, Stewart KG, Chai S, Manocha U, Byrd KM, Damrauer JS, Williams SE, Vincent BG, Kim WY: Molecular subtype-specific immunocompetent models of high-grade urothelial carcinoma reveal differential neoantigen expression and response to immunotherapy. Cancer Res 2018, 78:3954-3968

16. Fantini D, Glaser AP, Rimar KJ, Wang Y, Schipma M, Varghese N, Rademaker A, Behdad A, Yellapa A, Yu Y, Sze CC, Wang L, Zhao Z, Crawford SE, Hu D, Licht JD, Collings CK, Bartom E, Theodorescu D, Shilatifard A, Meeks JJ: A carcinogeninduced mouse model recapitulates the molecular alterations of human muscle invasive bladder cancer. Oncogene 2018, 37: $1911-1925$

17. Puzio-Kuter AM, Castillo-Martin M, Kinkade CW, Wang X, Shen TH, Matos T, Shen MM, Cordon-Cardo C, Abate-Shen C: Inactivation of $\mathrm{p} 53$ and Pten promotes invasive bladder cancer. Genes Dev 2009, 23:675-680

18. Owczarek TB, Kobayashi T, Ramirez R, Rong L, Puzio-Kuter AM, Iyer G, Teo MY, Sanchez-Vega F, Wang J, Schultz N, Zheng T, Solit DB, Al-Ahmadie HA, Abate-Shen C: ARF confers a context- dependent response to chemotherapy in muscle-invasive bladder cancer. Cancer Res 2017, 77:1035-1046

19. Nishizawa K, Nishiyama H, Matsui Y, Kobayashi T, Saito R, Kotani H, Masutani H, Oishi S, Toda Y, Fujii N, Yodoi J, Ogawa O: Thioredoxin-interacting protein suppresses bladder carcinogenesis. Carcinogenesis 2011, 32:1459-1466

20. Vasconcelos-Nobrega C, Colaco A, Lopes C, Oliveira PA: Review: BBN as an urothelial carcinogen. In Vivo 2012, 26:727-739

21. Shin K, Lim A, Zhao C, Sahoo D, Pan Y, Spiekerkoetter E, Liao JC, Beachy PA: Hedgehog signaling restrains bladder cancer progression by eliciting stromal production of urothelial differentiation factors. Cancer Cell 2014, 26:521-533

22. Van Batavia J, Yamany T, Molotkov A, Dan H, Mansukhani M, Batourina E, Schneider K, Oyon D, Dunlop M, Wu XR, CordonCardo C, Mendelsohn C: Bladder cancers arise from distinct urothelial sub-populations. Nat Cell Biol 2014, 16:982-991. 1-5

23. Tarutani M, Itami S, Okabe M, Ikawa M, Tezuka T, Yoshikawa K, Kinoshita T, Takeda J: Tissue-specific knockout of the mouse Pig-a gene reveals important roles for GPI-anchored proteins in skin development. Proc Natl Acad Sci U S A 1997, 94:7400-7405

24. Luche H, Weber O, Nageswara Rao T, Blum C, Fehling HJ: Faithful activation of an extra-bright red fluorescent protein in "knock-in" Cre-reporter mice ideally suited for lineage tracing studies. Eur J Immunol 2007, 37:43-53

25. Olive KP, Tuveson DA, Ruhe ZC, Yin B, Willis NA, Bronson RT, Crowley D, Jacks T: Mutant p53 gain of function in two mouse models of Li-Fraumeni syndrome. Cell 2004, 119:847-860

26. Shen TH, Gladoun N, Castillo-Martin M, Bonal D, DomingoDomenech J, Charytonowicz D, Cordon-Cardo C: A BAC-based transgenic mouse specifically expresses an inducible Cre in the urothelium. PLoS One 2012, 7:e35243

27. Ciardiello F, Caputo R, Bianco R, Damiano V, Pomatico G, De Placido S, Bianco AR, Tortora G: Antitumor effect and potentiation of cytotoxic drugs activity in human cancer cells by ZD-1839 (Iressa), an epidermal growth factor receptor-selective tyrosine kinase inhibitor. Clin Cancer Res 2000, 6:2053-2063

28. Liu L, Ye TH, Han YP, Song H, Zhang YK, Xia Y, Wang NY, Xiong Y, Song XJ, Zhu YX, Li de L, Zeng J, Ran K, Peng CT, Wei YQ, Yu LT: Reductions in myeloid-derived suppressor cells and lung metastases using AZD4547 treatment of a metastatic murine breast tumor model. Cell Physiol Biochem 2014, 33:633-645

29. Seager C, Puzio-Kuter AM, Cordon-Cardo C, McKiernan J, AbateShen C: Mouse models of human bladder cancer as a tool for drug discovery. Curr Protoc Pharmacol 2010. Chapter 14:Unit 14

30. R Development Core Team: R, A Language and Environment for Statistical Computing. Vienna, Austria, R Foundation for Statistical Computing, 2009

31. Ritchie ME, Phipson B, Wu D, Hu Y, Law CW, Shi W, Smyth GK: Limma powers differential expression analyses for RNA-sequencing and microarray studies. Nucleic Acids Res 2015, 43:e47

32. Bult CJ, Blake JA, Smith CL, Kadin JA, Richardson JE; Mouse Genome Database Group: Mouse genome database (MGD) 2019. Nucleic Acids Res 2019, 47:D801-D806

33. Jain AK, Dubes RC: Algorithms for Clustering Data. Englewood Cliffs, NJ, Prentice Hall Inc., 1988

34. Subramanian A, Tamayo P, Mootha VK, Mukherjee S, Ebert BL, Gillette MA, Paulovich A, Pomeroy SL, Golub TR, Lander ES, Mesirov JP: Gene set enrichment analysis: a knowledge-based approach for interpreting genome-wide expression profiles. Proc Natl Acad Sci U S A 2005, 102:15545-15550

35. Mootha VK, Lindgren CM, Eriksson KF, Subramanian A, Sihag S, Lehar J, Puigserver P, Carlsson E, Ridderstrale M, Laurila E, Houstis N, Daly MJ, Patterson N, Mesirov JP, Golub TR, Tamayo P, Spiegelman B, Lander ES, Hirschhorn JN, Altshuler D, Groop LC: PGC-1alpha-responsive genes involved in oxidative phosphorylation are coordinately downregulated in human diabetes. Nat Genet 2003, $34: 267-273$ 
36. Saito R, Shirakawa R, Nishiyama H, Kobayashi T, Kawato M, Kanno T, Nishizawa K, Matsui Y, Ohbayashi T, Horiguchi M, Nakamura T, Ikeda T, Yamane K, Nakayama E, Nakamura E, Toda Y, Kimura T, Kita T, Ogawa O, Horiuchi H: Downregulation of Ral GTPase-activating protein promotes tumor invasion and metastasis of bladder cancer. Oncogene 2013, 32:894-902

37. Warrick JI, Walter V, Yamashita H, Chung E, Shuman L, Amponsa VO, Zheng Z, Chan W, Whitcomb TL, Yue F, Iyyanki T, Kawasawa YI, Kaag M, Guo W, Raman JD, Park JS, DeGraff DJ: FOXA1, GATA3 and PPAR cooperate to drive luminal subtype in bladder cancer: a molecular analysis of established human cell lines. Sci Rep 2016, 6:38531

38. Kamoun A, de Reynies A, Allory Y, Sjodahl G, Robertson AG, Seiler R, Hoadley KA, Groeneveld CS, Al-Ahmadie H, Choi W, Castro MAA, Fontugne J, Eriksson P, Mo Q, Kardos J, Zlotta A, Hartmann A, Dinney CP, Bellmunt J, Powles T, Malats N, Chan KS, Kim WY, McConkey DJ, Black PC, Dyrskjot L, Hoglund M, Lerner SP, Real FX, Radvanyi F; Bladder Cancer Molecular Taxonomy Group: A consensus molecular classification of muscleinvasive bladder cancer. Eur Urol 2020, 77:420-433

39. Seiler R, Ashab HAD, Erho N, van Rhijn BWG, Winters B, Douglas J, Van Kessel KE, Fransen van de Putte EE, Sommerlad M, Wang NQ, Choeurng V, Gibb EA, Palmer-Aronsten B, Lam LL, Buerki C, Davicioni E, Sjodahl G, Kardos J, Hoadley KA, Lerner SP, McConkey DJ, Choi W, Kim WY, Kiss B, Thalmann GN, Todenhofer T, Crabb SJ, North S, Zwarthoff EC, Boormans JL, Wright J, Dall'Era M, van der Heijden MS, Black PC: Impact of molecular subtypes in muscleinvasive bladder cancer on predicting response and survival after neoadjuvant chemotherapy. Eur Urol 2017, 72:544-554
40. Seiler R, Gibb EA, Wang NQ, Oo HZ, Lam HM, van Kessel KE, Voskuilen CS, Winters B, Erho N, Takhar MM, Douglas J, Vakar-Lopez F, Crabb SJ, van Rhijn BWG, Fransen van de Putte EE, Zwarthoff EC, Thalmann GN, Davicioni E, Boormans JL, Dall'Era M, van der Heijden MS, Wright JL, Black PC: Divergent biological response to neoadjuvant chemotherapy in muscle-invasive bladder cancer. Clin Cancer Res 2019, 25:5082-5093

41. Yoshida T, Kates M, Fujita K, Bivalacqua TJ, McConkey DJ: Predictive biomarkers for drug response in bladder cancer. Int J Urol 2019, 26:1044-1053

42. Nickerson ML, Witte N, Im KM, Turan S, Owens C, Misner K, Tsang SX, Cai Z, Wu S, Dean M, Costello JC, Theodorescu D: Molecular analysis of urothelial cancer cell lines for modeling tumor biology and drug response. Oncogene 2017, 36:35-46

43. Gandhi D, Molotkov A, Batourina E, Schneider K, Dan H, Reiley M, Laufer E, Metzger D, Liang F, Liao Y, Sun TT, Aronow B, Rosen R, Mauney J, Adam R, Rosselot C, Van Batavia J, McMahon A, McMahon J, Guo JJ, Mendelsohn C: Retinoid signaling in progenitors controls specification and regeneration of the urothelium. Dev Cell 2013, 26:469-482

44. He X, Marchionni L, Hansel DE, Yu W, Sood A, Yang J, Parmigiani G, Matsui W, Berman DM: Differentiation of a highly tumorigenic basal cell compartment in urothelial carcinoma. Stem Cells 2009, 27:1487-1495

45. Warrick JI, Sjodahl G, Kaag M, Raman JD, Merrill S, Shuman L, Chen G, Walter V, DeGraff DJ: Intratumoral heterogeneity of bladder cancer by molecular subtypes and histologic variants. Eur Urol 2019, $75: 18-22$ 